\title{
Design and Realization of the Vibration Cylinder Barograph Data Acquisition System
}

\author{
Feng SHA, Chang-hao WANG, Hai-ming LI \\ The Test Center, Unit 91388,PLA, Zhanjiang, China \\ 38795490@qq.com, shallopfeng@live.com, 2005595@qq.com
}

\begin{abstract}
Data acquisition system is the core module of the automated calibration software. In this paper, a vibration cylinder barograph data acquisition system has been developed, applying LabVIEW. The development environment of hardware has been described. In process of the automated calibration, the system can achieve data acquisition, display and save functions. The system code has good extensibility, can be used in the development of other similar software. (Abstract)
\end{abstract}

Keywords-Calibration;Vibration Cylinder Barograph; LabVIEW; Data Acquisition System (key words)

\section{INTRODUCTION}

With the widespread use of measurement software, especially the automated calibration system, Metrology software plays increasingly important role in the development of modern equipment metrology technology. With the targets of shorten development cycle, improve usability and reliability, a vibration cylinder barograph automated calibration software has been developed, this paper mainly introduces its data acquisition system.

\section{THE BRIEF INTRODUCTION OF LABVIEW}

LabVIEW(Laboratory Virtual Instrument Engineering Workbench) is a innovative software product of National Instruments (NI). It is a powerful graphical software development integrated environment, being widely used and development speed rapidly, and being regarded as a standard data acquisition and instrument control software. The soft panel, which is developed with LabVIEW, has many advantages, just like interface Friendly, operate easily and intuitive, etc. LabVIEW is easy to learn and it can save a lot of development time and improve the efficiency of development, Compared with traditional programming languages.

\section{THE DEVELOPMENT ENVIRONMENT OF HARDWARE}

Vibration cylinder barograph data acquisition system is developed on the hardware platform of the pressure calibration standard device.

The hardware environment of vibration cylinder barograph calibration is consist of standard device and waiting devices.

The standard device of vibration cylinder barograph calibration is consist of special instrument calibration rack, automatic pressure controller, pressure standard device, labor controlling machine, positive pressure pump and vacuum pump.

Calibration standard device can remote control the automatic pressure controller through host computer, and can setup calibration parameters as calibration pressure point and the pressure change rate. Automatic pressure controller can control the pressure points automatically according to the configured parameters.

Calibration standard device also offer eight standard RS232 data interface, which are independent of each other, can be used for collecting the test data of the vibration cylinder barograph.

Labor controlling machine include host computer, data communication ports, etc. Operator can not only control automatic pressure controller, but also collect, record and process data through the host computer.

The special instrument calibration rack has 8 data communication cables and 8 pressure path plugs, which can meet the needs of the operator to calibrate different number of instruments. As shown in Figure 1.The DB9 RS232 communication interface of special instrument calibration rack connect the data interface of host computer through the DB62-pin extension cable, which can achieve real-time communication between waiting devices and host computer.

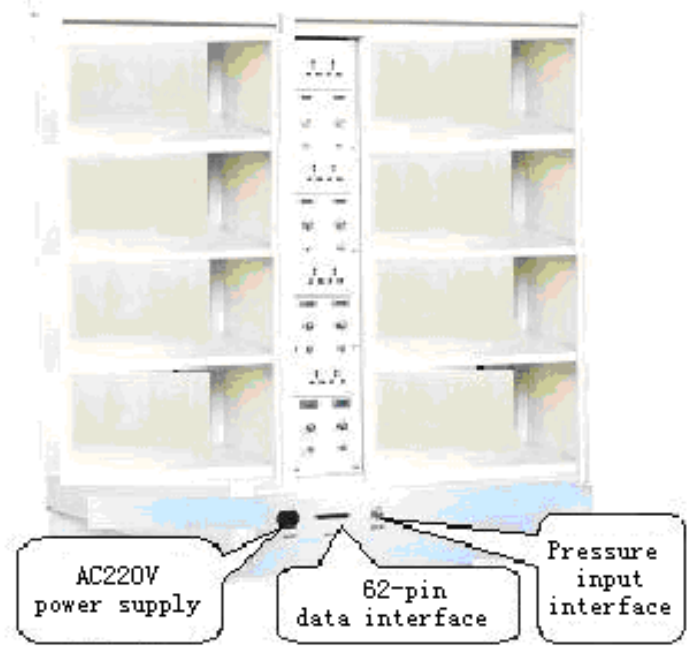

Figure 1. The special instrument calibration rack.

As the pressure standard device, digital barometer provides standard pressure values for the automatic controller in the calibration process.

The waiting device is vibration cylinder barograph, which has RS232 data interface.

\section{SOFTWARE DESIGN}

It can quickly achieve for data acquisition operations on the computer depend on the flowchart programming 
techniques and strong drivers of LabVIEW. Through data communication ports, the data of standard device and waiting devices are collected and displayed, the host computer will save and process the data.

Software system is modular in design, divided into three modules, shown in Figure 2.

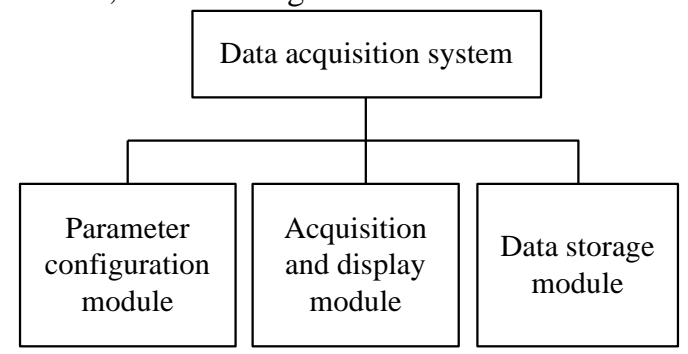

Figure 2. The components of data acquisition system

The main functions of parameter configuration module are to setup calibration points, the number of strokes, stability range, stability time, pressure speed, the environment, communications and other configuration parameters; According to the parameters configured, acquisition and display module collect and display the data of standard device and waiting devices real-time. Data storage module is responsible for saving the collected data. Data acquisition system flow chart is shown in Figure 3.

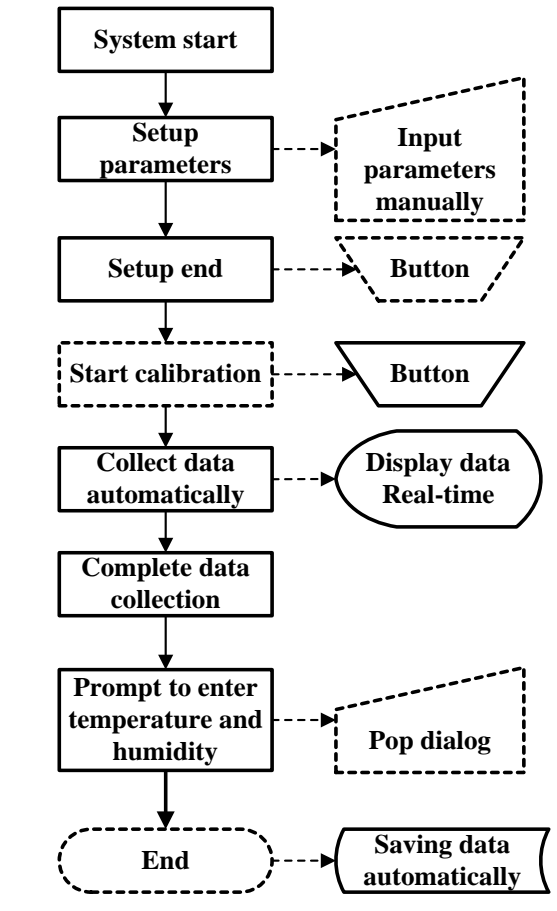

Figure 3. Data acquisition system flow chart
After start the system, a dialog box will pop to prompt the user to setup parameters. Click the button to complete the setup, and enter the calibration part. Click the start button, the system will collect and display the real-time data automatically. When all data collection are completed, a dialog box will pop to prompt the user to enter the temperature and humidity, the system will save the data automatically after entering, and finally the system will be stopped.

\section{A. Parameter configuration module}

\section{1) Calibration point}

In the allowable range, calibration point can be set in any value and has a pre-setting function.

Enter a value, sub-VI determines whether the value is in the allowable range, if it is, the value will be displayed; if not, a dialog box will pop to ask the user to re-enter. Results displayed are arranged by ascending.

\section{2) Lifting stroke}

The stroke is down then up, the number of strokes have three options " $1,2,4$."

"1" means down stroke only, calibration points are arranged by descending.

"2" means down then up stroke, calibration points are arranged by descending then ascending.

" 4 " means running " 2 " twice.

3) Stability parameters

Stability parameters include stability range and stability time.

Stability range is the limit fluctuations pressure when nearly stabilized, it can be setup, and the range is generally $\pm 0.1 \mathrm{hPa}$ for each calibration point.

Stability time is the time pressure maintained within the stability range at each calibration point, it can be setup, and the time is generally 2 minutes.

The stability lamp will light, when the difference changes between standard device and vibration cylinder barograph aren't more than $\pm 0.1 \mathrm{hPa}$ within 2 minutes at each calibration point.

4) Pressure speed

It is used to control the rate of device pressure change, and the speed is generally lower than $100 \mathrm{hPa} / \mathrm{min}$.

5) Environmental parameters 
Before and after the calibration, a dialog box pops up respectively to prompt the operator to input temperature and relative humidity.

6) Communication parameters
The host computer can display the real-time data of standard device and waiting devices.

The Block diagram of standard device communication and waiting device communication are shown in Figure 4 and Figure 5.

Standard device communication

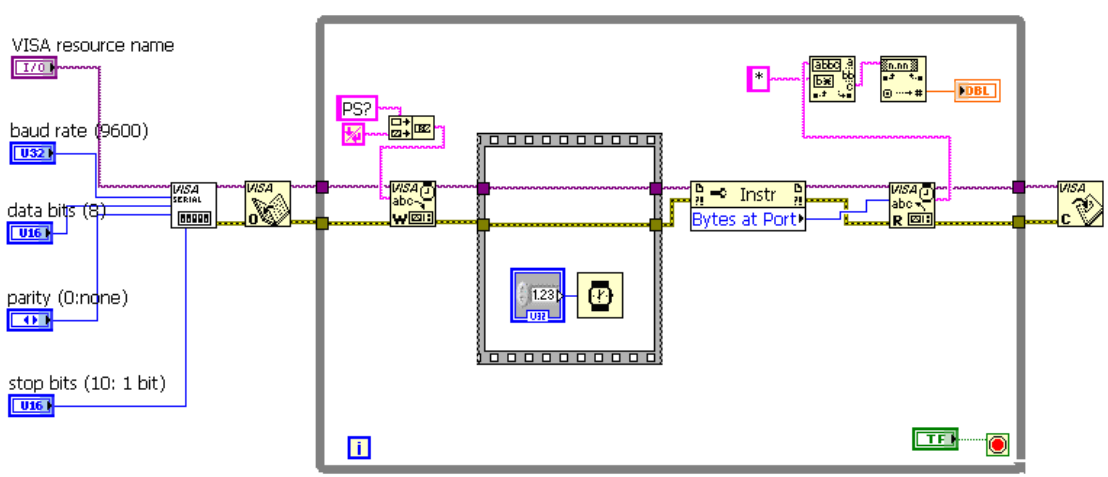

Figure 4. The Block diagram of standard device communication

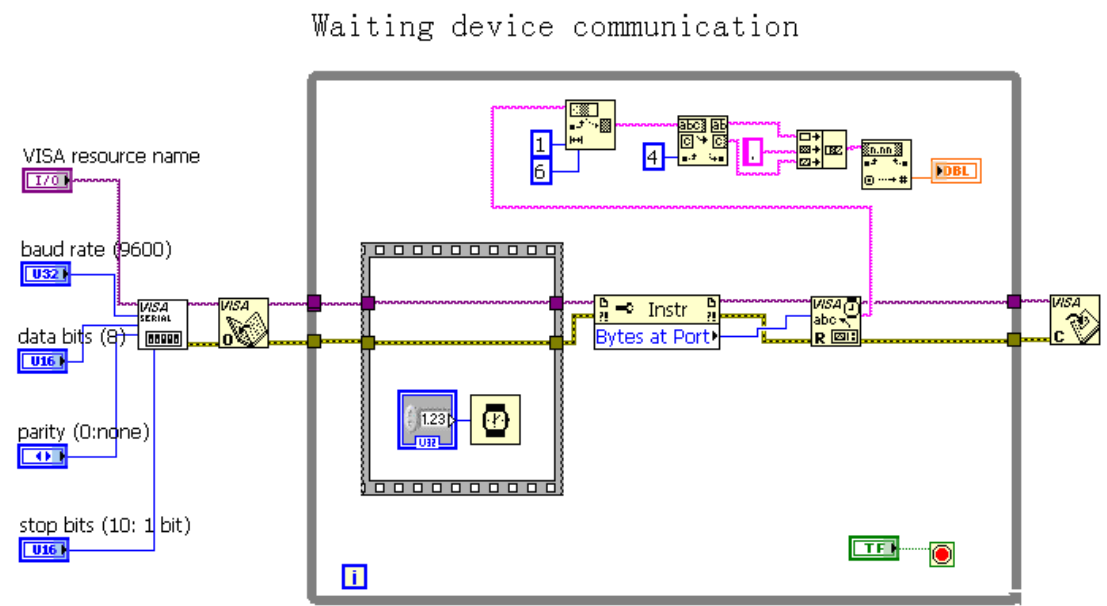

Figure 5. The Block diagram of waiting device communication

\section{B. Acquisition and display module}

The main functions of acquisition and display module are to collect and display the data of standard device, vibration cylinder barograph, each calibration point, stability time, and calibration time and so on.

After the start of the calibration, each calibration point and the real-time pressure of standard device and each channel vibration cylinder barograph are displayed. When the stability range of each calibration point is arrived, the stability time is counted and displayed. While the stability time is arrived, the lamp will light, at the same time the data of standard device and vibration cylinder barograph will be collected and displayed.

The Interface is designed, through numerical indicators (Liquid tanks) which display the real-time data of standard device and vibration cylinder barograph, and a table indicator which displays calibration points and each pressure collected from the standard device and the vibration cylinder barograph.

The software running flow chart of data Acquisition and display module is shown in Figure 6. 


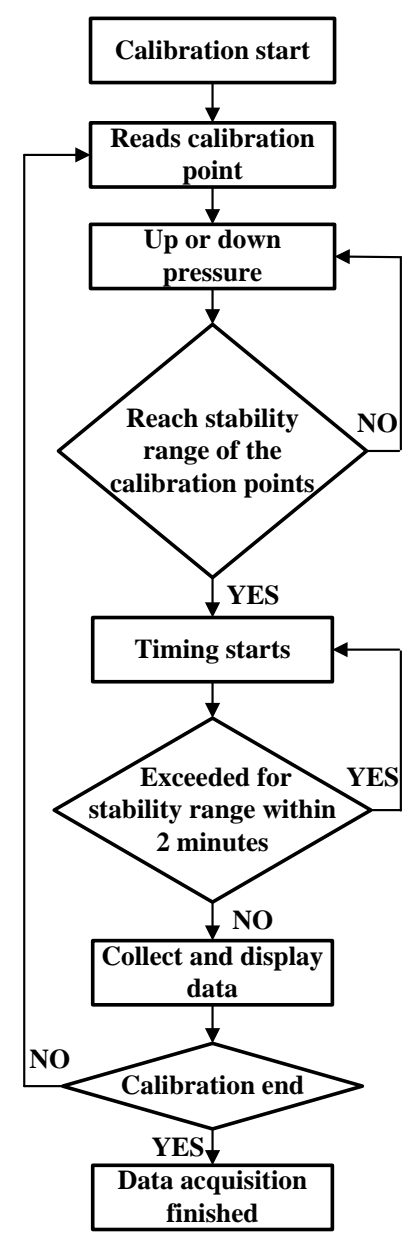

Figure 6. The software running flow chart of data Acquisition and display module

\section{Data save module}

The main function of data save module is to save the data, which is collected from standard device and waiting devices in each calibration point.

Each calibration point and the data collected are saved together in a Excel file which is named X years X months $\mathrm{X}$ day $\mathrm{X}$ hour $\mathrm{X}$ minute vibration cylinder barograph calibration data.

\section{The consolidated achieve of data acquisition functions}

Many VIs have been designed in parameter configuration module, acquisition and display module and data storage module. The integrated design of the data acquisition system can be finished by selecting the appropriate structure and interface, combining the functions of these VIs. As shown in Figure 7.

\section{SUMMARY}

The system has a beautiful interface, and easy to operate. Each design code features are designed into subVIs, which are very easy to maintain, in the development of data acquisition system. It has high degree of automation, operator just need setup parameters before calibration, the software will collect data in the process and stop automatically after calibration. In terms of reliability, it collects accurate data, runs stable and has a good fault tolerance. When the system is closed, it will return the pressure calibration standard to normal atmospheric first, before closing to ensure the safety of equipment. The codes of the software have good reusability, and ease to develop other calibration software which have similar functions.

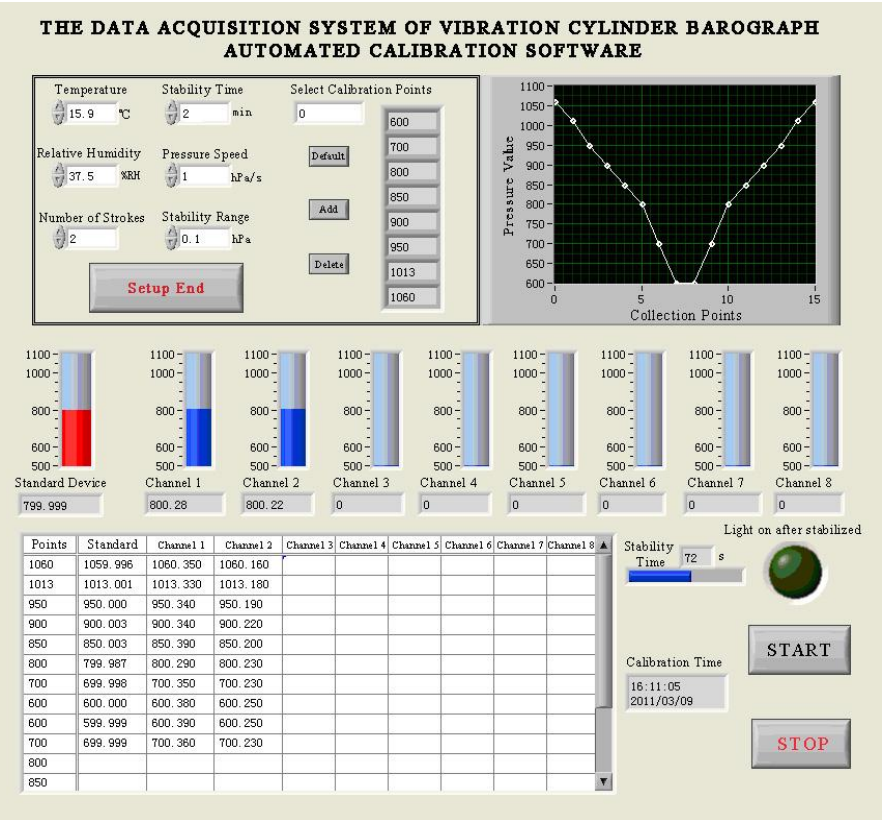

Figure 7. The data acquisition system of vibration cylinder barograph automated calibration software

\section{REFERENCES}

[1] Bolt A, Mazur G H. Integrating service and product quality function

deployment[EB/OL].http://www.mazur.net/works/Jurassic.qfd.pdf. ,2003-06-30.

[2] Terninko J. Step-by-Step QFD: Customer-driven product design (2nd ed) [M].Boca Raton, FL: St Lucie Press, 1997.3-6.

[3] Sullivan L P.Quality Function Deployment[J].Quality Progress, 1991,19(6):39-50.

[4] Crow K.Customer-Focused Development with QFD[EB/OL].http://www.Npd- solutions .com/qf-d.htm1.2000.

[5] Matzler K.Hinterhuber H H.How to make product development projects more successful by integrating Kano's model of customer satisfaction into quality function deployment[J].Technovation,1998,18(1):25-38.

[6] Customer Requirements Mapping Method Based on Association Rule Mining for Mass Customization[J].Shanghai Jiaotong Univ.(Sci.), 2008,13(3):291-296.

[7] Crow K.Customer-Focused Development with QFD[EB/OL].http://www.Npd- solutions .com/qf-d.htm1.2000.

[8] Crow K.Performing QFD step by step[EB/OL].http://www.npdsolutions. com/qfdstep6.htm.2000.

[9] Customer Requirements Mapping Method Based on Association Rule Mining for Mass Customization[J].Shanghai Jiaotong Univ.(Sci.), 2008,13(3):291-296.D. Kornack and P. Rakic, "Cell Proliferation without Neurogenesis in Adult Primate Neocortex," Science, vol. 294, Dec. 2001, pp. 2127-2130, doi:10.1126/science.1065467. 